\title{
Anhedonia and Dysphoria Are Differentially Associated with the Risk of Dementia in the Cognitively Normal Elderly Individuals: A Prospective Cohort Study
}

\author{
Ju Ri Lee', Seung Wan Suh², Ji Won Han', Seonjeong Byun ${ }^{3,4}$, Soon Jai Kwon ${ }^{5}$, Kyoung Hwan Lee ${ }^{6}$, \\ Kyung Phil Kwak', Bong Jo Kim ${ }^{8}$, Shin Gyeom Kim${ }^{9}$, Jeong Lan Kim ${ }^{10}$, Tae Hui Kim ${ }^{11}$, Seung-Ho Ryu' ${ }^{12}$, \\ Seok Woo Moon ${ }^{13}$, Joon Hyuk Park ${ }^{14}$, Dong-Woo Lee ${ }^{15}$, Jong Chul Youn ${ }^{16}$, Dong Young Lee ${ }^{4,17}$, \\ Seok Bum Lee ${ }^{18}$, Jung Jae Lee ${ }^{18}$, Jin Hyeong $\mathrm{Jhoo}^{19}$, and Ki Woong Kim ${ }^{1,4,20,21} \bowtie$ \\ ${ }^{1}$ Department of Neuropsychiatry, Seoul National University Bundang Hospital, Seongnam, Republic of Korea \\ ${ }^{2}$ Department of Psychiatry, Kangdong Sacred Heart Hospital, Hallym University College of Medicine, Seoul, Republic of Korea \\ ${ }^{3}$ Department of Neuropsychiatry, National Medical Center, Seoul, Republic of Korea \\ ${ }^{4}$ Department of Psychiatry, Seoul National University College of Medicine, Seoul, Republic of Korea \\ ${ }^{5}$ Department of Psychiatry, Mediplex Sejong Hospital, Incheon, Republic of Korea \\ ${ }^{6}$ Department of Psychiatry, Bongseng Memorial Hospital, Busan, Republic of Korea \\ ${ }^{7}$ Department of Psychiatry, Dongguk University Gyeongju Hospital, Gyeongju, Republic of Korea \\ ${ }^{8}$ Department of Psychiatry, Gyeongsang National University School of Medicine, Jinju, Republic of Korea \\ ${ }^{9}$ Department of Neuropsychiatry, Soonchunhyang University Bucheon Hospital, Bucheon, Republic of Korea \\ ${ }^{10}$ Department of Psychiatry, School of Medicine, Chungnam National University, Daejeon, Republic of Korea \\ ${ }^{11}$ Department of Psychiatry, Yonsei University Wonju Severance Christian Hospital, Wonju, Republic of Korea \\ ${ }^{12}$ Department of Psychiatry, School of Medicine, Konkuk University, Konkuk University Medical Center, Seoul, Republic of Korea \\ ${ }^{13}$ Department of Psychiatry, School of Medicine, Konkuk University, Konkuk University Chungju Hospital, Chungju, Republic of Korea \\ ${ }^{14}$ Department of Neuropsychiatry, Jeju National University Hospital, Jeju, Republic of Korea \\ ${ }^{15}$ Department of Neuropsychiatry, Inje University Sanggye Paik Hospital, Seoul, Republic of Korea \\ ${ }^{16}$ Department of Neuropsychiatry, Kyunggi Provincial Hospital for the Elderly, Yongin, Republic of Korea \\ ${ }^{17}$ Department of Neuropsychiatry, Seoul National University Hospital, Seoul, Republic of Korea \\ ${ }^{18}$ Department of Psychiatry, Dankook University Hospital, Cheonan, Republic of Korea \\ ${ }^{19}$ Department of Psychiatry, Kangwon National University School of Medicine, Chuncheon, Republic of Korea \\ ${ }^{20}$ Department of Brain and Cognitive Science, Seoul National University College of Natural Sciences, Seoul, Republic of Korea \\ ${ }^{21}$ National Institute of Dementia, Seongnam, Republic of Korea
}

Objective We investigated the impact of depressed mood (dysphoria) and loss of interest or pleasure (anhedonia)on the risk of dementia in cognitively-normal elderly individuals.

Methods This study included 2,685 cognitively-normal elderly individuals who completed the baseline and 4-year follow-up assessments of the Korean Longitudinal Study on Cognitive Aging and Dementia. We ascertained the presence of dysphoria and anhedonia using the Mini International Neuropsychiatric Inventory. We defined subjective cognitive decline as the presence of subjective cognitive complaints without objective cognitive impairments. We analyzed the association of dysphoria and anhedonia with the risk of cognitive disorders using multinomial logistic regression analysis adjusted for age, sex, education, Cumulative Illness Rating Scale score, Apolipoprotein E genotype, and neuropsychological test performance.

Results During the 4-year follow-up period, anhedonia was associated with an approximately twofold higher risk of mild cognitive impairment $(\mathrm{OR}=2.09,95 \% \mathrm{CI}=1.20-3.64, \mathrm{p}=0.008)$ and fivefold higher risk of dementia $(\mathrm{OR}=5.07,95 \% \mathrm{CI}=1.44-17.92, \mathrm{p}=0.012)$ but was not associated with the risk of subjective cognitive decline. In contrast, dysphoria was associated with an approximately twofold higher risk of subjective cognitive decline $(\mathrm{OR}=2.06,95 \% \mathrm{CI}=1.33-3.19, \mathrm{p}=0.001)$ and 1.7 -fold higher risk of mild cognitive impairment $(\mathrm{OR}=1.75,95 \% \mathrm{CI}=1.00-3.05, \mathrm{p}=0.048)$ but was not associated with the risk of dementia.

Conclusion Anhedonia, but not dysphoria, is a risk factor of dementia in cognitively-normal elderly individuals.

Psychiatry Investig 2019;16(8):575-580

Key Words Anhedonia, Dysphoria, Depression, Dementia.

Received: March 6, 2019 Accepted: June 7, 2019

$\triangle$ Correspondence: Ki Woong Kim, MD, PhD

Department of Neuropsychiatry, Seoul National University Bundang Hospital, 82 Gumi-ro 173beon-gil, Bundang-gu, Seongnam 13620, Republic of Korea

Tel: +82-31-787-7432, Fax: +82-31-787-4058, E-mail: kwkimmd@snu.ac.kr

(c) This is an Open Access article distributed under the terms of the Creative Commons Attribution Non-Commercial License (https://creativecommons.org/licenses/by-nc/4.0) which permits unrestricted non-commercial use, distribution, and reproduction in any medium, provided the original work is properly cited. 


\section{INTRODUCTION}

Although the association between depression and the risk of dementia has been reported in many previous cross-sectional and prospective studies, ${ }^{1}$ it was not replicated in some studies $^{2,3}$ and its underlying mechanisms remain controversial. Depression may represent a risk factor of dementia, ${ }^{4,5}$ a prodromal symptom of dementia, ${ }^{6-10}$ a reaction to the symptoms of dementia, ${ }^{11}$ or a comorbid disorder that shares common pathophysiology with dementia., ${ }^{1,12}$

To diagnose major or minor depressive disorders, the Diagnostic and Statistical Manual of Mental Disorders-Fourth Edition (DSM-IV) requires one of two core symptoms to be present; being depressed or sad (hereafter dysphoria) and having markedly decreased interest or pleasure (hereafter anhedonia). ${ }^{13}$ Although anhedonia is less recognized than dysphoria and often misunderstood as a part of aging process, ${ }^{14}$ it is more prevalent than dysphoria, ${ }^{15,16}$ and plays an increasingly important role in the presentation of depression in older adults. ${ }^{17-21}$ Furthermore, anhedonia is a key symptom of apathy. ${ }^{22}$ Apathy has been repeatedly identified as an early symptom linked to clinical progression in mild cognitive impairment (MCI) and Alzheimer's disease (AD), ${ }^{23}$ and was associated with the hypometabolism of posterior cingulate cortex..$^{24}$ In our previous prospective study, anhedonia, but not dysphoria, was associated with the risk of AD conversion in people with MCI. ${ }^{25}$

A recent study showed that apathy-anhedonia was associated with the neurodegeneration of brain regions related to $\mathrm{AD}$ in cognitively normal older adults. ${ }^{26}$ However, to our knowledge, whether dysphoria or anhedonia is associated with future risk of cognitive decline in cognitively-normal elderly individuals has not been investigated. In this community-based prospective cohort study, we investigated the differential impact of dysphoria and anhedonia on the risk of cognitive decline in the older adults with normal cognition.

\section{METHODS}

\section{Subjects}

This study was conducted as a part of the Korean Longitudinal Study on Cognitive Aging and Dementia (KLOSCAD) ${ }^{27}$ The KLOSCAD is a nationwide population-based prospective cohort study with elderly Koreans launched in November 2009. In the KLOSCAD, 6,818 individuals who were randomly sampled from residential rosters of 13 districts of Korea, were aged 60 or older, and spoke Korean participated in the baseline assessment and have been followed since then every 2 years.

In the current study, we included 4,471 cognitively-normal elderly individuals after excluding 101 participants with Axis
I disorders listed in the DSM-IV ${ }^{13}$ or neurologic disorders that could affect their cognitive function. Of them, 2,685 completed the 4-year follow-up assessments (Figure 1). The non-responders to the follow-up assessment were older $(\mathrm{p}<0.001)$ and less educated ( $\mathrm{p}<0.001$ ), had higher Cumulative Illness Rating Scale (CIRS) score ( $\mathrm{p}=0.028)$ and lower Korean version of Consortium to Establish a Registry for Alzheimer's Disease (CERAD-K) Neuropsychological Assessment Battery total score (CERADTS, $\mathrm{p}<0.001)$, and showed more dysphoria $(\mathrm{p}<0.001)$ and anhedonia $(\mathrm{p}<0.001)$ than the responders. However, sex and the presence of the APOE e4 allele were not significantly different between the two groups.

All participants provided written informed consent. This study was approved by the Institutional Ethics Review Board of Seoul National University Bundang Hospital (B-0912-089010).

\section{Assessments}

Geriatric neuropsychiatrists with expertise in dementia research administered a face-to-face standardized diagnostic interview, neurological and physical examinations, and laboratory tests using the CERAD-K Clinical Assessment Battery ${ }^{28}$ and the Korean version of the Mini International Neuropsy-

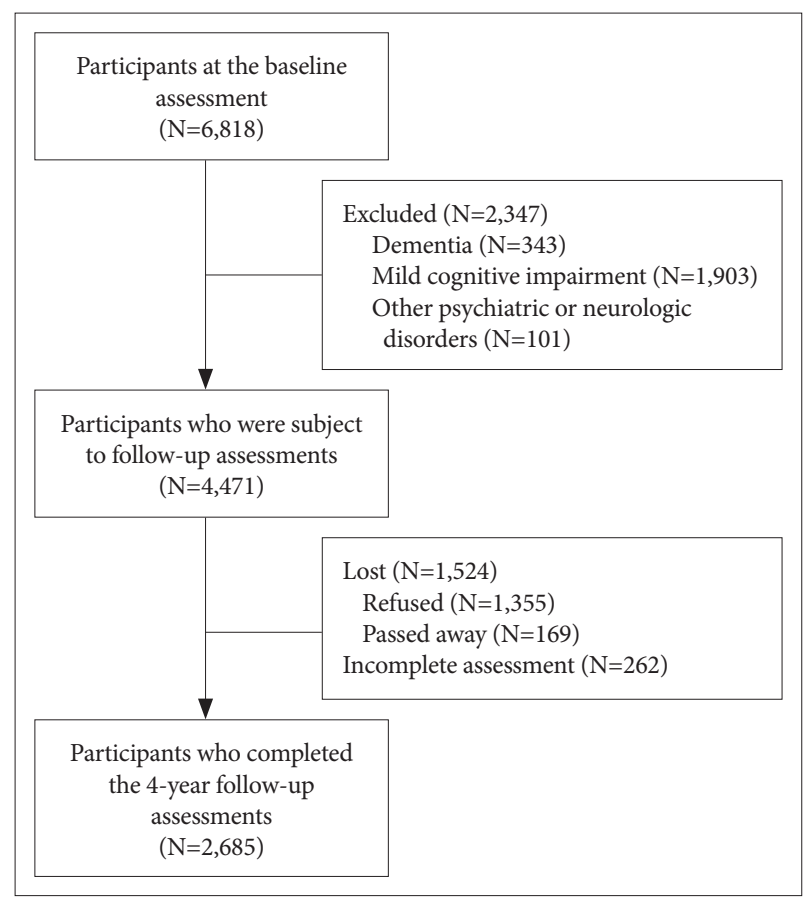

Figure 1. Study flowchart. At the baseline assessment, 6,818 individuals participated. We excluded participants who were diagnosed with dementia $(\mathrm{N}=343)$, mild cognitive impairment $(\mathrm{N}=$ $1,903)$, and 101 participants with other psychiatric or neurologic disorders $(\mathrm{N}=2,347)$. Among them 1,355 participants refused follow up assessment, 169 passed away before follow up, and 262 had incomplete assessment. A total of 2,685 participants completed the 4-year follow-up assessment. 
chiatric Inventory (MINI-K). ${ }^{29}$ They also evaluated comorbid medical conditions using the CIRS. ${ }^{30,31}$ Research neuropsychologists administered the CERAD-K Neuropsychological Assessment Battery, Lexical Fluency Test, ${ }^{32}$ and Digit Span Test ${ }^{33}$ to the subjects, to collect baseline and follow-up data. The CERADK-N consists of nine neuropsychological subtests, including the Categorical Fluency Test, the modified Boston Naming Test, the Mini-Mental State Examination (MMSE), the Word List Memory Test, the Constructional Praxis Test, the Word List Recall Test, the Word List Recognition Test, the Constructional Recall Test, and the Trail Making Test A. We obtained the CERAD-TS by summing the scores of the subtests of the CERAD-K Neuropsychological Assessment Battery except for the MMSE, Constructional Praxis Test, and Constructional Recall Test. ${ }^{34}$

We defined the presence of subjective cognitive complaints based on the clinical judgement of research geropsychiatrists. We ascertained the presence of objective cognitive impairment if a subject scored worse than -1.5 standard deviations (SD) on the age-, sex-, and education-adjusted norms for Korean elderly individuals on any of the 11 neuropsychological tests. We diagnosed dementia according to the DSM-IV diagnostic criteria ${ }^{13}$ and MCI according to the consensus criteria proposed by the International Working Group on MCI. ${ }^{35,36}$ We categorized the subjects who had subjective cognitive complaints but no objective cognitive impairment as subjective cognitive decline (SCD) and those who did not have both subjective cognitive complaints and objective cognitive impairments as cognitively normal (CN). We diagnosed major and minor depressive disorders according to the DSM-IV criteria. We defined dysphoria as the presence of depressed mood and anhedonia as lack of interest or pleasure for more than a half of day or more than seven days during the past two weeks.

\section{Statistical analysis}

To compare demographic characteristics between groups, we used Pearson's chi-square test for categorical variables and independent samples t-test for continuous ones. We used multinomial logistic regression analysis to examine the influence of baseline dysphoria and anhedonia on future cognitive decline in the $\mathrm{CN}$ group. In this analysis, the dependent variable was categorized into four groups; 1) remained as $\mathrm{CN}, 2$ ) progressed to SCD, 3) progressed to MCI, and 4) progressed to dementia. This model was adjusted for age, sex, education, CIRS score, CERAD-TS, and the presence of the apolipoprotein $\varepsilon 4$ allele. Statistical analyses were performed using SPSS 18.0 for Windows (SPSS Inc., Chicago, IL, USA).

\section{RESULTS}

Dysphoria and anhedonia were more prevalent in women at the baseline assessment $(\mathrm{p}<0.001)$. The participants with anhedonia at the baseline assessment were less educated than those without anhedonia ( $\mathrm{p}=0.028)$. However other baseline demographic and clinical characteristics did not differ based on the presence of dysphoria or anhedonia (Table 1).

Among the 2,685 CN participants who completed the 4-year follow-up assessment, 193 (7.2\%) and 183 (6.8\%) had dysphoria and anhedonia respectively at the baseline assessment. Among the 193 participants with dysphoria, $59(30.6 \%)$ progressed to SCD, 39 (20.2\%) to MCI, and eight (4.1\%) to dementia during the 4-year follow-up period. Among the 183 participants with anhedonia, 51 (27.9\%) progressed to SCD, 40 (21.9\%) to MCI, and nine $(4.9 \%)$ to dementia during the same follow-up period. In the multinomial logistic regression analysis, anhedonia was associated with an approximately two-fold higher risk of MCI (OR=2.089, 95\% CI=1.198-3.641, p=0.008) and approximately five-fold higher risk of dementia $(\mathrm{OR}=5.073,95 \%$ $\mathrm{CI}=1.436-17.922, \mathrm{p}=0.012)$ but was not associated with the risk of SCD $(\mathrm{OR}=1.462,95 \% \mathrm{CI}=0.921-2.319, \mathrm{p}=0.107)$. In contrast, dysphoria was associated with an approximately twofold higher risk of SCD (OR=2.058, 95\% CI=1.329-3.186,

Table 1. Baseline demographic and clinical characteristics of the participants

\begin{tabular}{|c|c|c|c|c|c|c|c|c|}
\hline \multirow{2}{*}{ Characteristics } & \multicolumn{4}{|c|}{ Dysphoria (depressed mood) } & \multicolumn{4}{|c|}{ Anhedonia (loss of interest or pleasure) } \\
\hline & Present & Absent & t or $\chi^{2}$ & $\mathrm{p}$ & Present & Absent & tor $\chi^{2}$ & $\mathrm{p}$ \\
\hline Number & 193 & 2492 & & & 183 & 2502 & & \\
\hline Age (years)* & $67.88 \pm 5.98$ & $68.30 \pm 5.72$ & -0.983 & 0.326 & $68.23 \pm 5.92$ & $68.27 \pm 5.72$ & -0.091 & 0.928 \\
\hline Woman, $\mathrm{N}(\%)^{\dagger}$ & $141(73.1)$ & $1347(54.1)$ & 26.184 & $<0.001$ & $131(71.6)$ & $1357(54.2)$ & 20.733 & $<0.001$ \\
\hline Education (years)* & $8.70 \pm 5.30$ & $9.35 \pm 5.28$ & -1.636 & 0.102 & $8.48 \pm 5.11$ & $9.36 \pm 5.29$ & -2.199 & 0.028 \\
\hline Apo E4 carrier, $\mathrm{N}(\%)^{\dagger}$ & $45(23.3)$ & $506(20.3)$ & 0.996 & 0.182 & $41(22.4)$ & $510(20.4)$ & 0.427 & 0.285 \\
\hline CIRS* & $1.49 \pm 0.47$ & $1.51 \pm 0.57$ & -0.493 & 0.623 & $1.55 \pm 0.46$ & $1.50 \pm 0.57$ & 1.444 & 0.150 \\
\hline CERAD-TS* & $58.33 \pm 9.59$ & $59.13 \pm 9.84$ & -1.089 & 0.276 & $57.92 \pm 9.41$ & $59.15 \pm 9.85$ & -1.642 & 0.101 \\
\hline
\end{tabular}

*independent sample t-test for continuous variables, ${ }^{\dagger}$ pearson's chi-squared $\left(\chi^{2}\right)$ test for categorical variables. CERAD-TS: Consortium to Establish a Registry for Alzheimer's Disease Neuropsychological Battery total score, CIRS: Cumulative Illness Rating Scale 
$\mathrm{p}=0.001)$ and approximately 1.7 -fold higher risk $\mathrm{MCI}(\mathrm{OR}=$ $1.748,95 \% \mathrm{CI}=1.004-3.045, \mathrm{p}=0.048$ ) but was not associated with the risk of dementia $(\mathrm{OR}=2.235,95 \% \mathrm{CI}=0.603-8.288$, $\mathrm{p}=0.229$ ) (Table 2).

\section{DISCUSSION}

In this study, dysphoria and anhedonia had differential impact on the future risk of cognitive decline in cognitively normal elderly individuals. Anhedonia was associated with the future risks of MCI and dementia but not with the risk of SCD while dysphoria was associated with the future risks of SCD and MCI but not with the risk of dementia.

Bäckman et al. ${ }^{37}$ reported that motivation-related, but not mood-related, symptoms of depression were associated with cognitive performance in clinically non-depressed and nondemented elderly individuals. Recently, Donovan et al. ${ }^{26}$ reported that subthreshold symptoms of depression were associated with AD biomarkers of neurodegeneration in older adults without overt cognitive impairment. In their study, higher apathy-anhedonia score, but not dysphoria score, was associated with lower cerebral glucose metabolism AD-related cortical regions. Marshall et al. ${ }^{38}$ reported that greater apathy was associated with higher amyloid burden in MCI. In a prospective study with non-demented older adults, the patients with incident $\mathrm{AD}$ had more depressive symptoms than the participants without dementia at baseline, and motivation-related symptoms such as lack of interest, loss of energy, and concentration difficulties were dominant at the preclinical stage of $\mathrm{AD}^{39}$

Older adults who have dysphoria without anhedonia may be better adapting to depression. Loss of interest in usual activities may lead to less physical activity and greater social iso- lation, both of which increase the risk for dementia. Anhedonia may lead to a self-reinforcing cascade of events resulting in continually worsening cognition and functioning. ${ }^{40-42}$ In addition, depression is commonly induced by cerebrovascular diseases in late life, ${ }^{43,44}$ and cerebrovascular diseases were more strongly associated with anhedonia than dysphoria. ${ }^{45}$ To sum up, anhedonia, even when present at a subclinical level, may increase the future risk of dementia possibly via both amyloiddependent and amyloid-independent processes.

In contrast to anhedonia, dysphoria was associated with the future risk of SCD and MCI but not with the risk of dementia, indicating that dysphoria may increase the risk of subjective cognitive complaints but not with that of objective cognitive impairments. The determinants of subjective cognitive complaints are complex. In elderly individuals, subjective memory complaints can be either realistic self-observations on cognitive decline or secondary symptoms to depression. ${ }^{46}$ Subjective cognitive complaints were found to be inconsistently related to current cognitive impairment but consistently related to depression and personality traits such as neuroticism. ${ }^{47}$ In a large cross-sectional study on community-dwelling adults without dementia, subjective cognitive complaints were more likely related to symptoms of depression than concurrent cognitive impairment. ${ }^{48}$ The subjects who most emphatically complained of memory disturbance had greater tendency toward somatic complaining, higher feelings of anxiety about their physical health, and more negative feelings of their own competence and capabilities than those who did not complain of memory deterioration associated with aging. ${ }^{49}$

This study has several limitations. First, demographic and clinical characteristics of the responders to the follow-up evaluation were different from those of the non-responders. Second, apathy was not evaluated. Third, we evaluated the pres-

Table 2. Associations of dysphoria (depressed mood) and anhedonia (loss of interest or pleasure) with the risks of cognitive disorders at the 4-year follow-up assessment*

\begin{tabular}{|c|c|c|c|c|c|c|c|c|c|}
\hline & \multicolumn{3}{|c|}{ SCD } & \multicolumn{3}{|c|}{ MCI } & \multicolumn{3}{|c|}{ Dementia } \\
\hline & OR & $95 \% \mathrm{CI}$ & $\mathrm{p}$ & OR & $95 \% \mathrm{CI}$ & $\mathrm{p}$ & OR & $95 \%$ CI & $\mathrm{p}$ \\
\hline Age & 1.032 & $1.012-1.053$ & 0.001 & 1.026 & $1.003-1.049$ & 0.029 & 1.145 & $1.075-1.219$ & $<0.001$ \\
\hline Female & 1.211 & $0.972-1.508$ & 0.088 & 1.228 & $0.921-1.639$ & 0.162 & 0.750 & $0.327-1.720$ & 0.497 \\
\hline Education & 0.983 & $0.819-1.178$ & 0.851 & 1.031 & $0.999-1.065$ & 0.060 & 1.016 & $0.929-1.112$ & 0.730 \\
\hline APOE $\& 4$ allele & 1.029 & $0.807-1.313$ & 0.816 & 0.921 & $0.670-1.267$ & 0.612 & 1.851 & $0.847-4.043$ & 0.123 \\
\hline CIRS & 0.983 & $0.819-1.178$ & 0.851 & 1.071 & $0.858-1.336$ & 0.547 & 1.178 & $0.619-2.240$ & 0.617 \\
\hline CERAD-TS & 1.033 & $1.018-1.047$ & $<0.001$ & 0.922 & $0.906-0.939$ & $<0.001$ & 0.907 & $0.863-0.953$ & $<0.001$ \\
\hline Dysphoria & 2.058 & $1.329-3.186$ & 0.001 & 1.748 & $1.004-3.045$ & 0.048 & 2.235 & $0.603-8.288$ & 0.229 \\
\hline Anhedonia & 1.462 & $0.921-2.319$ & 0.107 & 2.089 & $1.198-3.641$ & 0.008 & 5.073 & $1.436-17.922$ & 0.012 \\
\hline
\end{tabular}

${ }^{*}$ multinomial logistic regression analysis adjusting for age, sex, education, presence of the APOE $\varepsilon 4$ allele, CIRS score, and CERAD-TS at the baseline assessment. APOE: apolipoprotein, CERAD-TS: Consortium to Establish a Registry for Alzheimer's Disease Neuropsychological Assessment Battery total score, CI: confidence interval, CIRS: Cumulative Illness Rating Scale, MCI: mild cognitive impairment, OR: odds ratio, SCD: subjective cognitive decline 
ence of dysphoria and anhedonia for only 2 weeks prior to the assessment despite that these symptoms are episodic in nature. Despite these limitations, however, anhedonia is more likely to increase future risk of dementia than dysphoria in cognitively normal elderly individuals.

\section{Acknowledgments}

This work was supported by a Haesong Geriatric Psychiatry Research Fund from the Korean Mental Health Foundation.

\section{Conflicts of Interest}

The authors have no potential conflicts of interest to disclose.

\section{Author Contributions}

Conceptulization: Ki Woong Kim. Data curation: Ju Ri Lee, Ji Won Han. Formal Analysis \& Software \& Visualization \& Writing_original draft: Ju Ri Lee, Ki Woong Kim. Funding acquisition: Ki Woong Kim. Investigation \& Methodology \& Resources \& Writing-review \& editing: Ju Ri Lee, Seung Wan Suh, Ji Won Han, Seonjeong Byun, Soon Jai Kwon, Kyoung Hwan Lee, Kyung Phil Kwak, Bong Jo Kim, Shin Gyeom Kim, Jeong Lan Kim, Tae Hui Kim, Seung-Ho Ryu, Seok Woo Moon, Joon Hyuk Park, Dong-Woo Lee, Jong Chul Youn, Dong Young Lee, Seok Bum Lee, Jung Jae Lee, Jin Hyeong Jhoo, Ki Woong Kim. Project administration \& Supervision \& Validation: Ki Woong Kim. Ji Won Han.

\section{ORCID iDs}

$\begin{array}{ll}\text { Ki Woong Kim } & \text { https://orcid.org/0000-0002-1103-3858 } \\ \text { Ju Ri Lee } & \text { https://orcid.org/0000-0001-7631-4004 }\end{array}$

\section{REFERENCES}

1. Byers AL, Yaffe K. Depression and risk of developing dementia. Nat Rev Neurol 2011;7:323-331.

2. Landes AM, Sperry SD, Strauss ME. Prevalence of apathy, dysphoria, and depression in relation to dementia severity in Alzheimer's disease. J Neuropsychiatry Clin Neurosci 2005;17:342-349.

3. Panza F, Capurso C, D'Introno A, Colacicco AM, Zenzola A, Menga R, et al. Impact of depressive symptoms on the rate of progression to dementia in patients affected by mild cognitive impairment. The Italian Longitudinal Study on Aging. Int J Geriatr Psychiatry 2008;23:726734.

4. Lindsay J, Laurin D, Verreault R, Hébert R, Helliwell B, Hill GB, et al. Risk factors for Alzheimer's disease: a prospective analysis from the Canadian Study of Health and Aging. Am J Epidemiol 2002;156:445-453.

5. Tsuno N, Homma A. What is the association between depression and Alzheimer's disease? Expert Rev Neurother 2009;9:1667-1676.

6. Yaffe K, Blackwell T, Gore R, Sands L, Reus V, Browner WS. Depressive symptoms and cognitive decline in nondemented elderly women: a prospective study. Arch Gen Psychiatry 1999;56:425-430.

7. Ritchie K, Gilham C, Ledésert B, Touchon J, Kotzki PO. Depressive illness, depressive symptomatology and regional cerebral blood flow in elderly people with sub-clinical cognitive impairment. Age Ageing 1999;28:385-391.

8. Andersen K, Lolk A, Kragh-Sørensen P, Petersen NE, Green A. Depression and the risk of Alzheimer disease. Epidemiology 2005;16:233238.

9. Devanand DP, Sano M, Tang MX, Taylor S, Gurland BJ, Wilder D, et al. Depressed mood and the incidence of Alzheimer's disease in the elderly living in the community. Arch Gen Psychiatry 1996;53:175-182.

10. Dal Forno G, Palermo MT, Donohue JE, Karagiozis H, Zonderman $\mathrm{AB}$, Kawas $\mathrm{CH}$. Depressive symptoms, sex, and risk for Alzheimer's disease. Ann Neurol 2005;57:381-387.

11. Chen P, Ganguli M, Mulsant BH, DeKosky ST. The temporal relation- ship between depressive symptoms and dementia: a community-based prospective study. Arch Gen Psychiatry 1999;56:261-266.

12. Alexopoulos GS. Vascular disease, depression, and dementia. J Am Geriatr Soc 2003;51:1178-1180.

13. American Psychiatric Association. Diagnostic and Statistical Manual of Mental Disorders, Fourth Ed. Washington, DC: American Psychiatric Publishing; 1994.

14. Sarkisian CA, Hays RD, Berry SH, Mangione CM. Expectations regarding aging among older adults and physicians who care for older adults. Med Care 2001;39:1025-1036.

15. Gallo JJ, Anthony JC, Muthén BO. Age differences in the symptoms of depression: a latent trait analysis. J Gerontol 1994;49:251-264.

16. Caine ED, Lyness JM, King DA, Connors L. Clinical and Etiological Heterogeneity of Mood Disorders in the Elderly. In: Schneider LS, Reynolds CF, Lebowitz B, Fiedhoff A, Editors. Diagnosis and Treatment of Depression in Late Life: Results of the NIH Consensus Development Conference. Washington DC: American Psychiatric Press, 1994, p.21-54.

17. Koenig HG, Blazer DG. Depression, Anxiety, and Other Mood Disorders. In: Cassel CK, Leipzig RM, Cohen HJ, Larson EB, Meier DE, Editors. Geriatric Medicine: An Evidence-based Approach. 4th Edition. New York: Springer-Verlag, 2003, p.1163-1183.

18. Ballard C, Bannister C, Solis M, Oyebode F, Wilcock G. The prevalence, associations and symptoms of depression amongst dementia sufferers. J Affect Disord 1996;36:135-144.

19. Chemerinski E, Petracca G, Sabe L, Kremer J, Starkstein SE. The specificity of depressive symptoms in patients with Alzheimer's disease. AmJPsychiatry 2001;158:68-72.

20. Janzing JGE, Hooijer C, van't Hof MA, Zitman FG. Depression in subjects with and without dementia: a comparison using GMS-AGECAT. Int J Geriatr Psychiatry 2002;17:1-5.

21. Lopez OL, Becker JT, Sweet RA, Klunk W, Kaufer DI, Saxton J, et al. Psychiatric symptoms vary with the severity of dementia in probable Alzheimer's disease. J Neuropsychiatry Clin Neurosci 2003;15:346353.

22. Robert PH, Berr C, Volteau M, Bertogliati C, Benoit M, Sarazin M, et al. Apathy in patients with mild cognitive impairment and the risk of developing dementia of Alzheimer's disease: a one-year follow-up study. Clin Neurol Neurosurg 2006;108:733-736.

23. Palmer K, Di Iulio F, Varsi AE, Gianni W, Sancesario G, Caltagirone C, et al. Neuropsychiatric predictors of progression from amnestic-mild cognitive impairment to Alzheimer's disease: the role of depression and apathy. J Alzheimers Dis 2010;20:175-183.

24. Delrieu J, Desmidt T, Camus V, Sourdet S, Boutoleau-Bretonnière C, Mullin E, et al. Apathy as a feature of prodromal Alzheimer's disease: an FDG-PET ADNI study. Int J Geriatr Psychiatry 2015;30:470-477.

25. So Y, Kim KW, Park JH, Lee JJ, Lee SB, Kim T. Anhedonia is associated with the risk of Alzheimer's disease in elders with mild cognitive impairment: results from the Korean Longitudinal Study on Health and Aging (KLOSHA). Alzheimers Dement 2012;8:594.

26. Donovan NJ, Hsu DC, Dagley AS, Schultz AP, Amariglio RE, Mormino EC, et al. Depressive symptoms and biomarkers of Alzheimer's disease in cognitively normal older adults. J Alzheimers Dis 2015;46:63-73.

27. Kim TH, Park JH, Lee JJ, Jhoo JH, Kim BJ, Kim JL, et al. Overview of the Korean longitudinal study on cognitive aging and dementia. Alzheimers Dement. 2013;9:626-627.

28. Lee JH, Lee KU, Lee DY, Kim KW, Jhoo JH, Kim JH, et al. Development of the Korean Version of the Consortium to Establish a Registry for Alzheimer's Disease Assessment Packet (CERAD-K) clinical and neuropsychological assessment batteries. J Gerontol B Psychol Sci Soc Sci 2002;57:47-53.

29. Yoo SW, Kim YS, Noh JS, Oh KS, Kim CH, NamKoong K, et al. Validity of Korean version of the mini-international neuropsychiatric interview. Anxiety Mood 2006;2:50-66.

30. Linn BS, Linn MW, Gurel L. Cumulative illness rating scale. J Am Geri- 
atr Soc1968;16:622-626.

31. Miller MD, Paradis CF, Houck PR, Mazumdar S, Stack JA, Rifai AH, et al. Rating chronic medical illness burden in geropsychiatric practice and research: application of the Cumulative Illness Rating Scale. Psychiatry Res 1992;41:237-248.

32. Ruff RM, Light RH, Parker SB, Levin HS. Benton controlled oral word association test: Reliability and updated norms. Arch Clin Neuropsychol 1996;11:329-338.

33. Wechsler D. Wechsler Memory Scale Revised. New York: The Psychological Corporation; 1987.

34. Chandler MJ, Lacritz LH, Hynan LS, Barnard HD, Allen G, Deschner $\mathrm{M}$, et al. A total score for the CERAD neuropsychological battery. Neurology 2005;65:102-106.

35. Petersen RC. Mild cognitive impairment as a diagnostic entity. J Intern Med 2004;256:183-194.

36. Winblad B, Palmer K, Kivipelto M, Jelic V, Fratiglioni L, Wahlund LO, et al. Mild cognitive impairment-beyond controversies, towards a consensus: report of the International Working Group on Mild Cognitive Impairment. J Intern Med 2004;256:240-246.

37. Bäckman L, Hill RD, Forsell Y. The influence of depressive symptomatology on episodic memory functioning among clinically nondepressed older adults. J Abnorm Psychol 1996;105:97-105.

38. Marshall GA, Donovan NJ, Lorius N, Gidicsin CM, Maye J, Pepin LC, et al. Apathy is associated with increased amyloid burden in mild cognitive impairment. J Neuropsychiatry Clin Neurosci 2013;25:302-307.

39. Berger AK, Fratiglioni L, Forsell Y, Winblad B, Bäckman L. The occurrence of depressive symptoms in the preclinical phase of $\mathrm{AD}$ : a population-based study. Neurology 1999;53:1998-2002.

40. Laurin D, Verreault R, Lindsay J, MacPherson K, Rockwood K. Physical activity and risk of cognitive impairment and dementia in elderly persons. Arch Neurol 2001;58:498-504.

41. Fratiglioni L, Wang HX, Ericsson K, Maytan M, Winblad B. Influence of social network on occurrence of dementia: a community-based longitudinal study. Lancet 2000;355:1315-1319.

42. Wang HX, Karp A, Winblad B, Fratiglioni L. Late-life engagement in social and leisure activities is associated with a decreased risk of dementia: a longitudinal study from the Kungsholmen project. Am J Epidemiol 2002;155:1081-1087.

43. Alexopoulos GS, Meyers BS, Young RC, Campbell S, Silbersweig D, Charlson M. 'Vascular depression' hypothesis. Arch Gen Psychiatry 1997;54:915-922.

44. Park JH, Lee SB, Lee JJ, Yoon JC, Han JW, Kim TH, et al. Epidemiology of MRI-defined vascular depression: a longitudinal, community-based study in Korean elders. J Affect Disord 2015;180:200-206.

45. Krishnan KR, Hays JC, Blazer DG. MRI-defined vascular depression. Am J Psychiatry 1997;154:497-501.

46. Schmand B, Jonker C, Geerlings MI, Lindeboom J. Subjective memory complaints in the elderly: depressive symptoms and future dementia. Br J Psychiatry 1997;171:373-376.

47. Reid LM, MacLullich AM. Subjective memory complaints and cognitive impairment in older people. Dement Geriatr Cogn Disord 2006; 22:471-485.

48. Zlatar ZZ, Moore RC, Palmer BW, Thompson WK, Jeste DV. Cognitive complaints correlate with depression rather than concurrent objective cognitive impairment in the successful aging evaluation baseline sample. J Geriatr Psychiatry Neurol 2014;27:181-187.

49. Hänninen T, Reinikainen KJ, Helkala EL, Koivisto K, Mykkänen L, Laakso M, et al. Subjective memory complaints and personality traits in normal elderly subjects. J Am Geriatr Soc 1994;42:1-4. 